\title{
Routing Optimization Algorithm of Spatial DTN Network Based on Multiattribute Decision
}

\author{
Hua Liang, ${ }^{1}$ Yang Yang $\mathbb{D}^{2}$ and Ziyan Wang ${ }^{1}$ \\ ${ }^{1}$ School of Information and Mechatronics Engineering, Hunan International Economics University, Changsha, \\ 410205 Hunan, China \\ ${ }^{2}$ School of Electronics and Information, Nanchang Institute of Technology, Nanchang, 330044 Jiangxi, China
}

Correspondence should be addressed to Yang Yang; zyn@nut.edu.cn

Received 5 August 2021; Revised 31 August 2021; Accepted 6 September 2021; Published 26 September 2021

Academic Editor: Zhihan Lv

Copyright () 2021 Hua Liang et al. This is an open access article distributed under the Creative Commons Attribution License, which permits unrestricted use, distribution, and reproduction in any medium, provided the original work is properly cited.

Delay or stop (DTN) tolerance space network is considered to be a technology that can trade with a limited or restricted field communication area. In the space DTN network architecture, routing is very important. This article is based on the best-inclass multiattribute decision-making DTN network routing optimization algorithm. The goal is to use the optimized DTN network routing algorithm multiattribute decision-making algorithm and conduct a more in-depth study on the field information mechanism multipath decision algorithm and network path. Aiming at the problems of long link delay and frequent link interruption of current deep-space communication characteristics, and solving the impact on communication caused by system performance degradation. This paper adopts the multiattribute decision-making model algorithm to construct simulation tests, introduces the DTN network architecture, and analyzes the four attributes of the multi-attribute decision-making link bandwidth, node data forwarding rate, link establishment delay, and network error rate. The experimental results show that, in the spatial information network, the high-speed movement of nodes and the highly dynamic nature of the network make the deep-space network topology unstable and long communication delay; the change of the link mode and switch connection in the network will cause the routing table to update. This routing update method requires the exchange of topology information between all networks; when the forwarding rates of adjacent nodes are $100 \%, 90 \%, 95 \%$, and $70 \%$, nodes can only perform routing independently when they meet. The decision attribute algorithm has obvious changes to the attributes of any node in the spatial DTN network. The most obvious is that the link establishment delay has reached the standard of 5 or more.

\section{Introduction}

With the development of satellite communication technology towards broadcasting and all-IP, satellite mobile communication technology is trying to integrate the design of terrestrial cellular networks to combine its most useful features [1]. This is an important trend in the development of next-generation mobile communication technology. Due to differences in space technology, system architecture, communication capabilities, and commercial support of various wireless communication networks, customized space technology mobile terminals cannot meet user requirements for one or another space technology, such as navigation service continuity and network power adaptation. With the development of science and technology, the Shenzhou, Sky-1, and Beidou series satellites have been continuously sent into space, and the structure of my country's space information network has been gradually improved. One of the development goals of my country's space information technology is to achieve full-time, full-coverage continuous communication, and expand human activities to space, oceans, and even deep space. The communication system is the key to the successful completion of deep space missions $[2,3]$.

The spatial information network is a comprehensive information network that integrates air, space, and ground resources [4]. Continuous communication and monitoring capabilities are important means and ways of real-time 
acquisition and interconnection of spatial information resources [5]. The frequent interruptions of links in the space DTN network, long delays, highly asymmetric data rates, high bit error rates in the space network environment, limited node resources, and limited life span make the routing algorithm of the space satellite DTN network and the ground network. The routing algorithm is very different, and because of the regularity of the node movement in the space satellite network, the routing algorithm can use the time-varying characteristics of the satellite network to make routing selection. Therefore, the optimal DTN network routing algorithm based on multidecision is an important research content. Therefore, the optimal DTN network routing algorithm based on multidecision is an important research content.

At present, many aspects of research on space information technology have been initiated at home and abroad, a lot of work has been done in the development of network architectures and protocols for satellite/space communications and interplanetary networks, and certain achievements have been made. Shi et al. have proposed a variety of solutions and have conducted a large number of literature surveys on these technologies. The delay/interruption tolerant network (DTN) architecture is proposed to implement automated network communications, despite the long link delays and frequent link interruptions that are usually characteristic of deep space communications. DTN is currently considered to be the only candidate protocol that is close to the maturity level required to handle the inevitable long link delays, frequent and lengthy link disconnections, and the massive data loss inherent in space communications [6]. $\mathrm{Yu}$ et al. focuses on network assessment and protocol deployment of complex deep space networks based on DTN and applies the results to a new complex deep space network based on the Universal Interplanetary Communication Network (UNICON-CDSN) proposed by the National Space Administration Science Center (NSSC) for simulation and verification. A network evaluation method based on network capacity and memory analysis is proposed. Based on the performance comparison of Licklider Transmission Protocol (LTP) and Transmission Control Protocol (TCP) and Bundling Protocol (BP) in various communication scenarios, a transmission protocol configuration scheme is proposed to construct UNICON's LTP deployment schemeCDSN. For the LTP deployment plan, a theoretical model of the file transfer time on the complex deep space network was established. Using the method proposed in this article for network evaluation, it is proved that UNICON-CDSN meets the requirements of Curiosity on Mars exploration mission in 2020. In addition, the simulation results of the Universal Space Communication Network Test Bed (USCNT) designed by us show that the LTP deployment scheme is suitable for UNICON-CDSN [7]. In Wang et al.'s research, delay tolerant network (DTN) is considered to be one of the most suitable technologies for handling challenging spacecraft communications. Routing is essential in the DTN architecture. Contact Graph Routing (CGR) is a dynamic system that calculates routes through the timevarying topology of predetermined communication links in the DTN architecture. There is an urgent need to study the scalability of CGR to very large networks. After discussing the operation of CGR, we proposed the performance evaluation of CGR on time-varying topologies of various scales. The purpose is to understand its availability in a spacecraft communication network with a large number of nodes [8]. They have conducted research on different aspects of spatial DTN network in terms of network protocol, network link, connection road map, etc. However, they still have shortcomings and lack of relevant research on the optimization algorithm of network routing, which cannot provide literature for this field supplement or reference value for more research. The multiattribute decision-making routing algorithm can analyze different fields such as network protocols and network links, and this method is more comprehensive.

Multiattribute decision making has applications in many fields. Especially in recent years, more and more decisionmaking methods have been used in actual evaluation and decision-making to judge and select weight attributes. The standardization method of decision-making attributes, the determination method of attribute weights, sensitivity analysis, and the application of multiattribute decision-making methods can provide a variety of values for the research of spatial DTN networks $[9,10]$. As an important part of the space-earth integrated network, satellite networks are naturally also the focus of scientific research in our country. With powerful and flexible global communication capabilities that are beyond the reach of terrestrial networks, satellite networks have rapidly developed into a research hotspot in modern communications. This paper analyzes the spatial DTN network routing algorithm based on multiattribute decision-making. Aiming at the network congestion problem caused by the existing DTN routing algorithm when multiple services are concurrent, using the time-varying graph theory, design the DTN network routing algorithm based on service perception, from the spatial DTN, the method of network routing and multiattribute decisionmaking is discussed, which has certain research value in the field of spatial DTN network routing.

\section{Multiattribute Decision-Making and Spatial DTN Network Algorithm}

2.1. Network Routing Algorithm Analysis. Routers are generally considered to be the most interesting and challenging content in this field [11]. Tracking is a choice of sending opportunities. It realizes the successful transmission of data to the last interface, which increases the possibility of increased transmission and reduces transmission delay. Routing algorithms have always been the research hotspot of DTN networks, and researchers at home and abroad have put forward many new research results. Satellite network communication system, using artificial earth satellites as space relay nodes, built in outer space, can provide full coverage of the earth's surface, is almost free from geographical factors, and will not be affected by natural disasters to a large extent Therefore, satellite networks have become the main trend in the development of communication networks. With network applications, products, and services as the core, the 
world's leading DNT network method based on multigeneration decision-making has become a hot spot in information network research. In the face of complex and robust network systems, existing routing algorithms cannot support high-bandwidth routing in uncertain environments and cannot guarantee efficient performance and improved resource utilization [12]. Therefore, based on the verification process, intelligent methods are adopted to solve the routing problem, which opens up new strategies for the routing research of the new generation information network.

The basic mode of network routing is "forward-forward" [13]. Therefore, the routing problem can be transformed into a problem of finding a "proactive" suitable meeting. Routing is a basic network function, including routing and data forwarding. In a traditional TCP/IP network, the router can build a routing table according to the current network topology information and write the next hop IP and corresponding data forwarding in the routing table output port; when the data packet arrives at the input port, it searches the routing table according to the destination address in the data packet header, finds the corresponding routing entry, and then forwards the data packet to the corresponding output port of the router [14]. Changes in the link status and link on-off relationship in the network will update the routing table. This routing update method requires the exchange of topology information across the entire network. Therefore, in network communication, it is necessary to ensure that there is a stable and continuous connection between nodes. At the same time, the network protocol has a relatively large overhead and a slower convergence speed. In the DTN network, the propagation time of loop communication is given by delayed signal transmission and frequent but short network intervals (i.e., interruptions). Even if the network transmission delay is small, unpredictable network conference movement can cause communication interruption. The optical network of the information network is owned by the satellite, and the path of the satellite is controlled and predictable. Therefore, when designing routing algorithms, we can use this prior knowledge to improve the efficiency of the algorithms, such as virtual topology routing algorithms, browser type platforms, and topologyrelated algorithms [15].

2.2. Method Analysis of Multiattribute Decision Making. Multiattribute decision-making is the research and analysis of multiattribute decision-making problems based on the decision maker's reference point, evidence reasoning, and prospect theory. Finally, the schemes are ranked according to the prospect value of each scheme [16]. At present, the application of multiattribute decision-making uses information entropy and gray correlation analysis to comprehensively evaluate multiattribute decision-making problems whose evaluation values are interval intuitionistic fuzzy numbers. Information entropy makes the original information more complete, and gray correlation analysis determines the degree of relevance of the plan. Through the power average operator, the binary linguistic mean function and the possibility degree ranking formula are given, and a multiattribute decision-making method based on entropy weight is proposed [17]. Assuming $a$, a certain satellite node has a data forwarding request, and there are satellite parts $m$ nearby to choose from. It is currently used as a data transmission page, that is, $m$ data transmission options are provided for the $L$ data source interface at this time. If each alternative has four attributes: link bandwidth, node data forwarding rate, link establishment delay, and network error rate, then an $m * 4$ multiattribute decision matrix can be established.

$$
a=\left\{\begin{array}{llll}
k_{1} & j_{1} & y_{1} & w_{1} \\
k_{2} & j_{2} & y_{2} & w_{2} \\
k_{3} & j_{3} & y_{3} & w_{3} \\
k_{4} & j_{4} & y_{4} & w_{4}
\end{array}\right\} .
$$

Each alternative has four attributes: different link bandwidth, node data forwarding rate, link establishment delay, and network error rate. According to the different attributes, multiple attributes are used to synthesize and evaluate the evaluation value range [18].

$$
\begin{gathered}
E_{a}=\sum_{l=1}^{m} \gamma_{l} \delta_{a l}, \\
H_{l a}=\frac{m_{l a}}{\sqrt{\sum_{l=1}^{m} h_{l a}}} \cdot \min _{k j y w} .
\end{gathered}
$$

In formula (2), $\delta_{a l}$ is the evaluation value of the evaluation value, and $\gamma_{l}$ is the standardized value; formula (2) is the formula for calculating the range of each attribute of each alternative, where $\min _{k j y w}$ is the best value of each attribute, that is, the best attribute of a single data node in the alternatives. However, for the selection of the commensurability problem between the attributes of the normalized value, the following formula is used for calculation.

$$
\begin{aligned}
Q_{l a} & =\frac{\left(1 / \varepsilon_{l a}\right) / \max _{m \in L}}{\min _{k j y w \notin H} / \varepsilon_{l a}}+1 . \\
q_{k j w y} & =\sum_{\varepsilon_{l a}}^{l=1}\left(\frac{a \bullet H_{\max }^{\min }}{1 / m \notin a}\right) .
\end{aligned}
$$

$q_{k j w y}$ means that a single attribute is selected for the commensurability of alternatives in the standardized numerical interval, and $\varepsilon_{l a}$ is the attribute weight value. Using the weight value to determine the ideal attributes and negative ideal attributes of the alternatives can be expressed as:

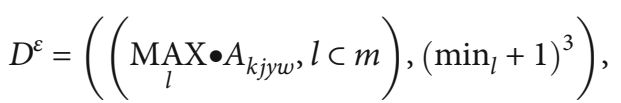




$$
\begin{gathered}
\left.D^{-\varepsilon}=\left(\min _{l} \bullet A_{k j w y},-1\right), \sum_{l=1} a \supset A_{m} m l \mid \varepsilon\right), \\
G_{l}=\frac{D_{L A}}{D^{\varepsilon}+D^{-\varepsilon}} \sum_{i_{o}} l^{i}-1 .
\end{gathered}
$$

$A_{k j y w}$ for each weight value, the ideal attribute of the alternative can be obtained by calculating the balance between the maximum normalized numerical interval and the lowest normalized numerical interval of the attribute $D^{\varepsilon}$. The negative ideal attribute $D^{-\mathcal{E}}$ of the alternative, ideal attribute, and negative, the ideal attribute is the decisionmaking process based on multiple attributes [19]. If $D^{\varepsilon}$ is closer to $D^{-\varepsilon}$, the $G_{l}$ is closer to 1 .

2.3. Spatial DTN Network. Through the operating environment of the spatial information network, we know that the spatial link has the characteristics of intermittent connectivity and long delay [20]. The introduction of satellites into the wireless network can effectively make up for the lack of coverage of the existing terrestrial network and provide users with ubiquitous voice, video and other real-time services, and broadband access services. Therefore, space has become an important area of development and exploration human being in the 21st century [21]. The ability to acquire and apply spatial information reflects a country's scientific and technological strength and economic development level and can meet the huge needs of economic construction, national security, scientific and technological development, and social development. It is an important feature of a country's comprehensive strength. The spatial DTN network has no end-to-end path, which is similar to a point spreading around, and a network is formed in space [22]. With the continuous expansion of the network scale and the rapid increase in the number of subnets, the current global optimal traditional routing algorithm will face serious challenges. The routing algorithm for multiattribute decisionmaking requires global maintenance of topology information. Each node only needs to maintain local topology information, but nodes cannot choose the local routing information that they are responsible for maintaining. Calculate all based on the distance between the source node and destination node names on the virtual ring, the probability of possible paths is calculated by calculating the average number of hops between nodes on the virtual ring, and finally, the actual path length is estimated. This will increase the time and difficulty of data processing to a certain extent. In addition, the spatial DTN network has the characteristics of high delay, low data transmission rate, limited node resources, and high error rate. Therefore, the purposeful use of these characteristics can greatly improve the current spatial information technology in my country. The link characteristics of the spatial DTN network can be expressed as:

$$
\frac{R_{l}}{S_{0}}=m_{i=1} \bullet \frac{t_{i}}{L_{\text {space }}} \cdot \frac{1}{L_{\text {pace }}} \bullet \frac{1}{q}\left(T_{i}, T_{i+1}\right)
$$

When a network data source node is

$$
R_{i=1}=\frac{T_{i=1}}{L_{\text {space }}} \bullet \frac{1}{L_{\text {pace }}}\left\{q \in \varepsilon, s=i^{m}\right\} .
$$

$L_{\text {space }}$ represents the nearest two data source nodes between a certain distance of the network link [23], $L_{\text {pace }}$ is the delay speed transmission rate of the data source node in the spatial DNT network, when the data transmission rate is greater, the link characteristic of the spatial DNT network will be smaller, but its value will not be 0 [24].

The bit error rate characteristics of the spatial DTN network can be calculated with the following formula [25]:

$$
\begin{gathered}
W_{F \in \delta}=p_{e} \int \frac{1}{2} \bullet \sqrt{f_{0}}-\left(l^{i-1} \sum_{e}^{i}\left(e+\min _{i}\right),\right. \\
w_{i}=\frac{\left(e / \sum_{i=1}^{1} f_{i+1}\right) e s c}{\gamma_{i=0}} .
\end{gathered}
$$

Calculate the size of the bit error rate by $W_{f \in \mathcal{E}}, P_{e}$ belongs to the size of the carrier frequency, and $\sqrt{f_{o}}$ is the wavelength between any two data sources in the spatial DTN network [26]. And the bit error rate of the spatial DTN network is determined by the wavelength and frequency. The relationship is that the longer the wavelength, the lower the carrier frequency, and the lower the bit error rate; conversely, the shorter the wavelength, the higher the carrier frequency and the lower the bit error rate [27].

Node resource constraints can be evaluated based on a multiattribute decision matrix, for example, based on the order of the matrix [28].

$$
\begin{gathered}
v_{\max }=\frac{\sum_{i=1} m \bullet \sum_{i=0}^{l} v_{i}^{q}}{m v^{i=0}}, \\
v_{0}=\left\{\sqrt{p_{e}}+\int\left(f_{0} \frac{i-1}{m}\right)-\sum_{\varepsilon^{i-1}}^{l} \gamma\right\} .
\end{gathered}
$$

Node resource limitation is an important attribute in multiattribute decision-making, which affects the overall calculation and technology application of spatial DTN network [29].

\section{Preparation Work of DTN Space Network}

\subsection{Object}

(1) Application of DTN protocol system and DTN network

(a) Deep space exploration

(b) Land mobile

(c) Military wireless self-organizing network space information network is not only for civilian use; it also has a wide range of military uses 


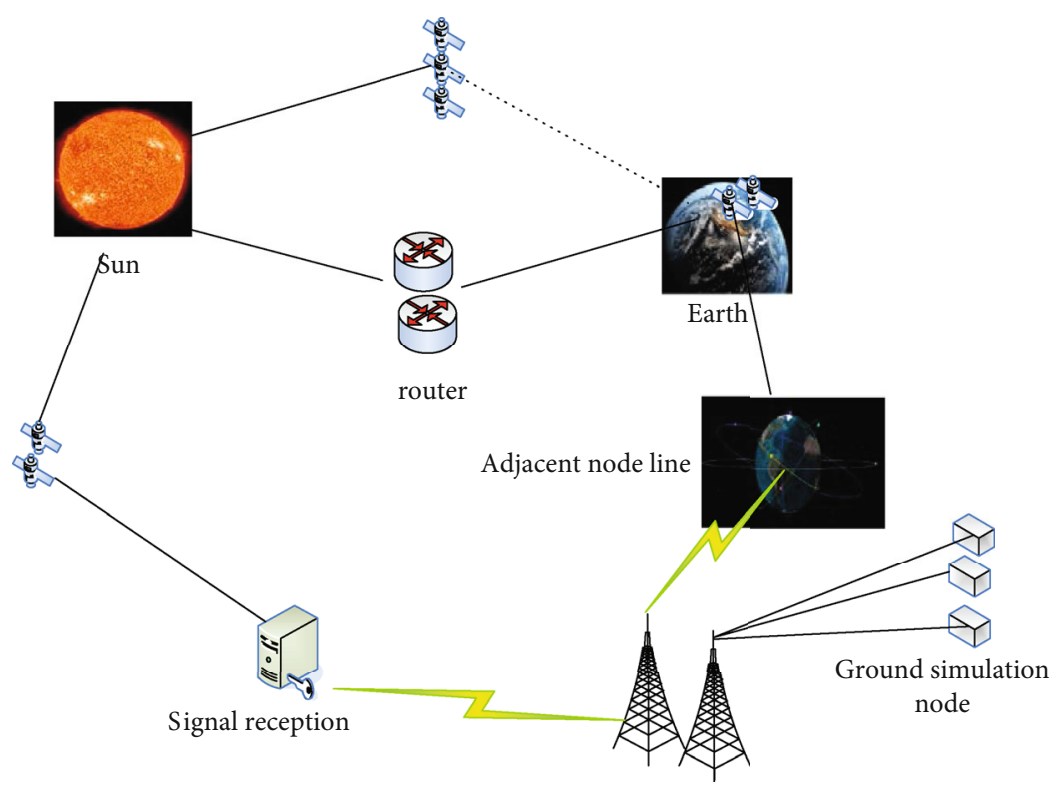

Figure 1: Simulation diagram of satellite in low true orbit.

and is a symbol of a country's aviation strength. Therefore, all countries will vigorously develop space information networks. For now, the spatial information network is still in the preliminary research stage

(2) Optimization of spatial DTN network based on multiattribute features

(3) Routing algorithm

\subsection{Process}

(1) The traditional network information system cannot be applied to the DTN network due to the high delay, frequent network delay, and multiple conversation sources of the DTN network; introduces the link characteristics of the spatial information network and the DTN network structure system

(2) The DTN network architecture is introduced. The DTN architecture adds a layer between the fivelayer application layer and the transport layer of the traditional network architecture to determine the forward transfer function in the network transmission process to adapt to the characteristics of the DTN network

(3) The current research status of DTN routing protocol and its application is introduced, including routing protocols based on multiattribute decision-making, and a comparative analysis of these routing information is carried out

(4) Change the forwarding method of messages in the search domain, reduce the copy of messages in the network, and achieve the purpose of saving network resources and improving network utilization
TABLe 1: Performance simulation analysis table.

\begin{tabular}{lcc}
\hline Category & $\begin{array}{c}\text { Network transmission } \\
\text { success rate }\end{array}$ & $\begin{array}{c}\text { Average network } \\
\text { delay }\end{array}$ \\
\hline Epidemic & 0.7 & 0.75 \\
PROPHED & 0.79 & 0.72 \\
No preference & 0.82 & 0.71 \\
$\begin{array}{l}\text { Forwarding rate } \\
\text { preference }\end{array}$ & 0.83 & 0.72 \\
Delay preference & 0.87 & 0.69 \\
\hline
\end{tabular}

\subsection{Results}

(1) Data transmission problem under the conditions of high bit error rate, long delay, uplink and downlink asymmetry, and frequent link interruption in the space network is solved

(2) Overall network analysis of multicomponent delay selective routing is slightly lower than that of nonselective multirouting algorithm

(3) The network transmission success rate of the nonpreference routing algorithm is lower than that of the routing algorithm with the routing rate preference; the average retention rate of the nonpreference routing algorithm is slightly higher than that of the multibinding routing algorithm

\section{Multiattribute Decision-Making Spatial DTN Network Routing Optimization Analysis}

4.1. Spatial DTN Network. The space communication network is a wireless communication network composed of multiple satellites or spacecraft. These satellites or spacecraft are usually distributed in different orbits because of their 


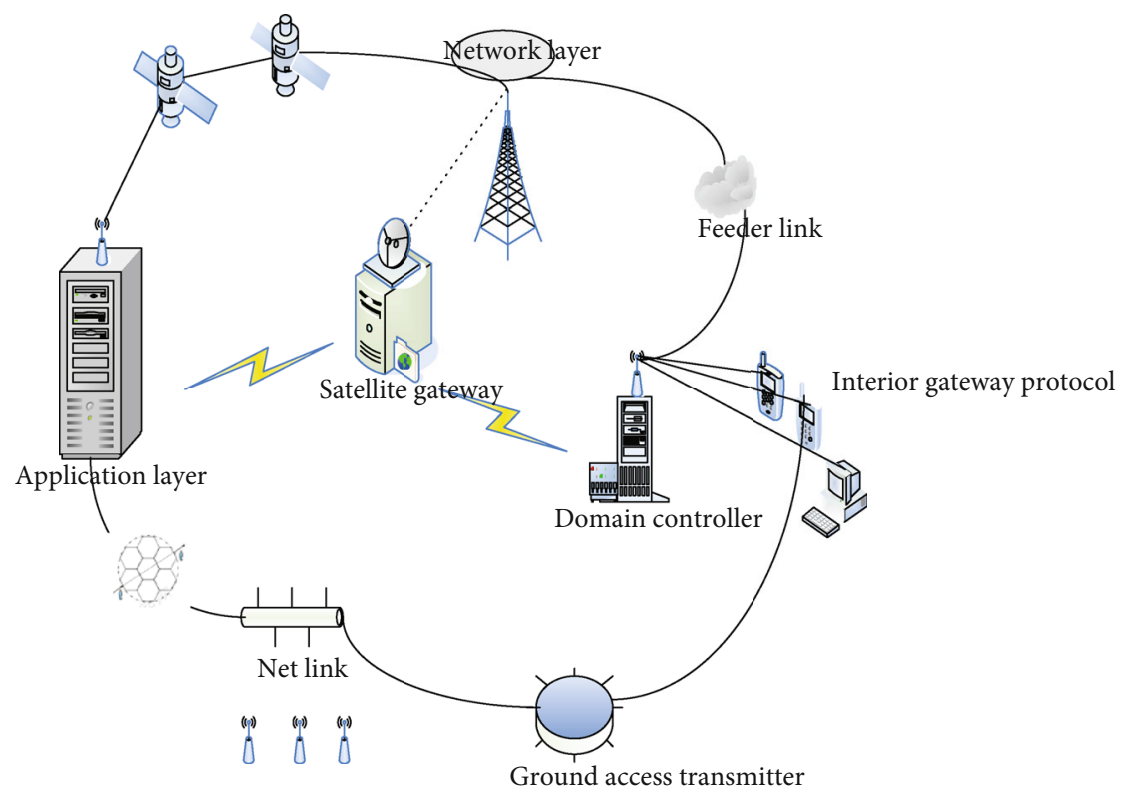

FIGURE 2: Link diagram of spatial DTN network structure.

different functional properties. The spatial communication network can realize the long-distance information transmission to verify the performance of the DTN network routing algorithm in the actual scene, design and realize the DTN network routing algorithm simulation data, and combine the routing algorithm designed in this paper with multiattribute decision-making for simulation experiments. In addition, this paper designs a test scenario based on the application of low-orbit satellites and builds a simulation platform Figure 1. This simulation platform mainly simulates the spatial DTN network through a variety of routing algorithms. DTN is a data packet layer added above the transmission layer and below the application layer of many different types of networks. A node can represent a specific space vehicle, and an edge can represent the link established between two space vehicles. The simulation function of the DTN network routing algorithm and the nature of the routing algorithm are run on the simulation platform. The periodic motion of the planet may block or interfere with the transmission of signals. In the spatial information network, the nodes move at high speed, and the network presents a high degree of dynamics, which makes the deep space network topology unstable and long communication delay.

In the space network environment, the size of the spacecraft, the power it carries, and the storage and computing resources are very limited. The limitation of various nodes will affect the transmission performance of the aerospace communication network, and it is impossible to obtain certain information about the movement of nodes in the network through prediction. The main difference between qualitative routing algorithms and random routing algorithms is that deterministic routing must be predictable. Table 1 is a table of various attribute parameters about network nodes.

The data in the above table shows that the overall network analysis of multicomponent delay selective routing is
TABLE 2: Spatial information network structure system.

Application layer (NTP protocol, DNT protocol, CFDP protocol, FTP protocol...)

Cover layer (BP protocol)

Licklider layer

Transport layer (TCP, UDP)

Network layer (IP)

Network interface layer (SONET, CCSDS)

slightly lower than that of nonselective multirouting algorithms. The network transmission success rate of the nonpreference routing algorithm is lower than that of the routing algorithm with the routing rate preference; the average retention rate of the nonpreference routing algorithm is slightly higher than that of the multibinding routing algorithm. Such a network environment can enable the DTN routing protocol to give full play to its advantages, and we need to improve the success rate of data delivery, while reducing network load and reducing network resource consumption. Figure 2 and Table 2, respectively, introduce the DTN network structure system and spatial information network link characteristics.

The router and the ground node carry out signal transmission and then generate software and evaluation software to evaluate the network node signal cooperation. The downlink is used to send data information, and the uplink is used to send control commands. This makes the probability of end-to-end path interruption higher. For real-time service transmission, the network performance is reduced due to link interruption, because the size of the control command relative to the data packet is small. In order to save the transmission power and bandwidth resources, the network performance can be reduced. The uplink rate is set to a lower value. 
4.2. Multiattribute Decision-Making Algorithm. Multidecision refers to the decision-making problem of choosing the best plan or classifying the plan in consideration of multiple factors. It is an important part of modern decision-making. Its ideas and methods are widely used in many fields such as technology, engineering, programming, management, and military. In recent years, the unpredictable multigenerational decision-making problem has attracted widespread attention. Due to the complexity and variability of objective conditions, the uncertainty of conceptual thinking, measurement errors, and other factors, the decision-making of the world's DTN network path is often complicated in form, which is an important theoretical average and relatively high material value. When selecting the optimal alternative, we used different network types for research. Table 3 shows the parameter table of the candidate network.

The parameters of the $3 \mathrm{G}$ cellular and satellite networks are displayed in the data in the above table. The magnitude of jitter is five levels of 1, 2, 3, 4, and 5. The larger the value, the greater the absolute value of the delay difference during forwarding. The user's expenditure in accessing the network is also described by five levels $(1,2,3,4$, and 5). Similarly, the larger the value the more the user access to the network. Although the position distribution of nodes in the network changes at all times, by knowing the position of each node at some initial moments and the orbital parameters of all nodes, it is possible to calculate the exact position of each node at any time. The movement of the node is actually predictable. According to the multidecision attribute algorithm in the second part of this article, we perform simulation calculations on the attributes of adjacent nodes. After the simulation experiment, the following data is obtained as shown in Figure 3, and the data Table 4 of related network nodes is attached.

In Figure 3 above, in order to more intuitively express the application of the multiattribute decision-making algorithm in the spatial DTN network, the link width, link establishment delay, and remaining storage space are all processed in accordance with a certain ratio of parameter data, al. The forwarding rates of the 8 adjacent nodes of a2, a3, a4, a5, a6, a7, and a8 are $100 \%, 90 \%, 95 \%$, and $70 \%$, respectively. Therefore, nodes can only perform routing selection independently when they meet, including routing algorithms based on message diffusion, multicopy routing based on control, and routing algorithms based on historical information. The algorithm complexity is as low as possible to save node energy. Through the statistical data analysis of the above figure, the multidecision attribute algorithm has obviously changed the attributes of any node in the spatial DTN network. The most obvious is that the link establishment delay has reached the standard of 5 or more. In a network with more predictable connections, the higher the data delivery rate that can be achieved by deterministic methods, the less bandwidth and cache space resources are wasted.

By analyzing the characteristics of the DTN network and the characteristics of the satellite network as one of the actual application scenarios, the defects of the existing routing algorithms suitable for traditional wireless networks and DTN are elaborated, and the ideal is determined according
TABle 3: Candidate network parameter list.

\begin{tabular}{lcc}
\hline \multirow{2}{*}{ Parameter name } & \multicolumn{2}{c}{ Parameter value } \\
& 3G cellular & Satellite \\
\hline Base station carrier frequency & $2 \mathrm{GHz}$ & $1.6 \mathrm{GHz}$ \\
Transmit power & $40 \mathrm{dBm}$ & $79 \mathrm{dBW}$ \\
Beam radius & $1.25 \mathrm{~km}$ & $125 \mathrm{~km}$ \\
Number of base stations & 7 & 3 \\
Transmission delay & $200 \mathrm{~ms}$ & $300 \mathrm{~ms}$ \\
Jitter level & 4 & 3 \\
Network fee & 2 & 4 \\
\hline
\end{tabular}

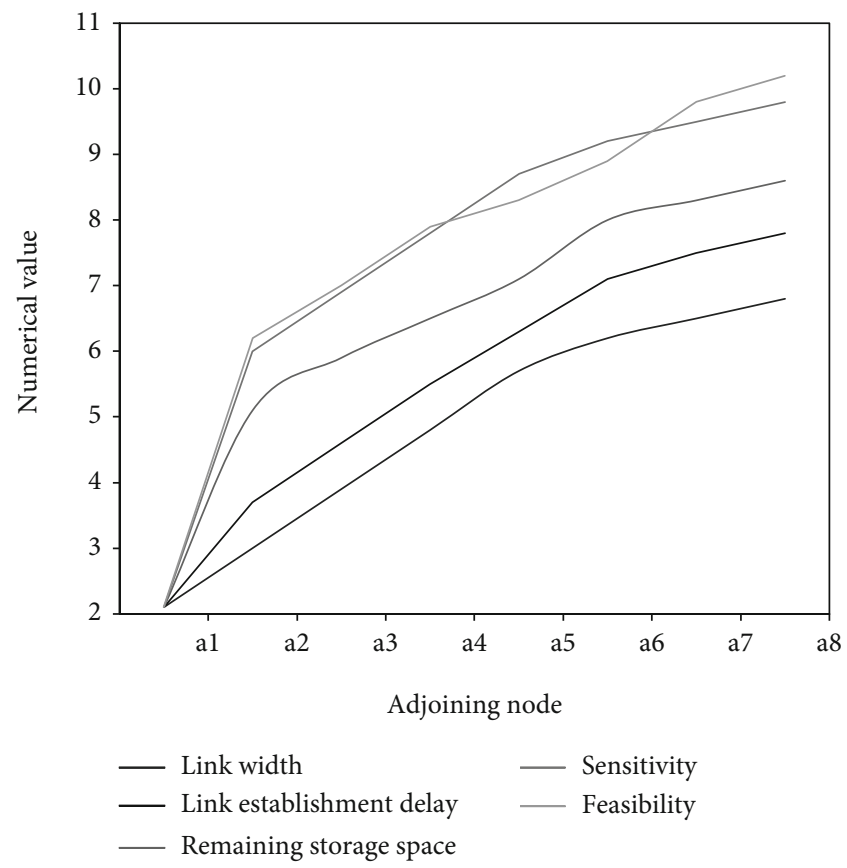

Figure 3: Simulation parameter data statistics table of adjacent nodes.

TABLE 4: Network node information configuration table.

\begin{tabular}{lccc}
\hline & $\begin{array}{c}\text { Orbital } \\
\text { period }\end{array}$ & $\begin{array}{c}\text { Orbital } \\
\text { inclination }\end{array}$ & $\begin{array}{c}\text { Ascending node } \\
\text { ascension }\end{array}$ \\
\hline $\begin{array}{l}\text { Satellite } \\
1\end{array}$ & 5400 & 28.5 & 10 \\
$\begin{array}{l}\text { Satellite } \\
2\end{array}$ & 5760 & 90 & 20 \\
Satellite & 7200 & 135 & 30 \\
3 & & & \\
Satellite & 17280 & 20 & 60 \\
4 & & & 70 \\
Satellite & 21600 & 0 & \\
5 & & & \\
\hline
\end{tabular}

to the weight value of the multiattribute decision-making algorithm. Alternatives, detailed analysis and classification are given, which provide a reference basis for future research 


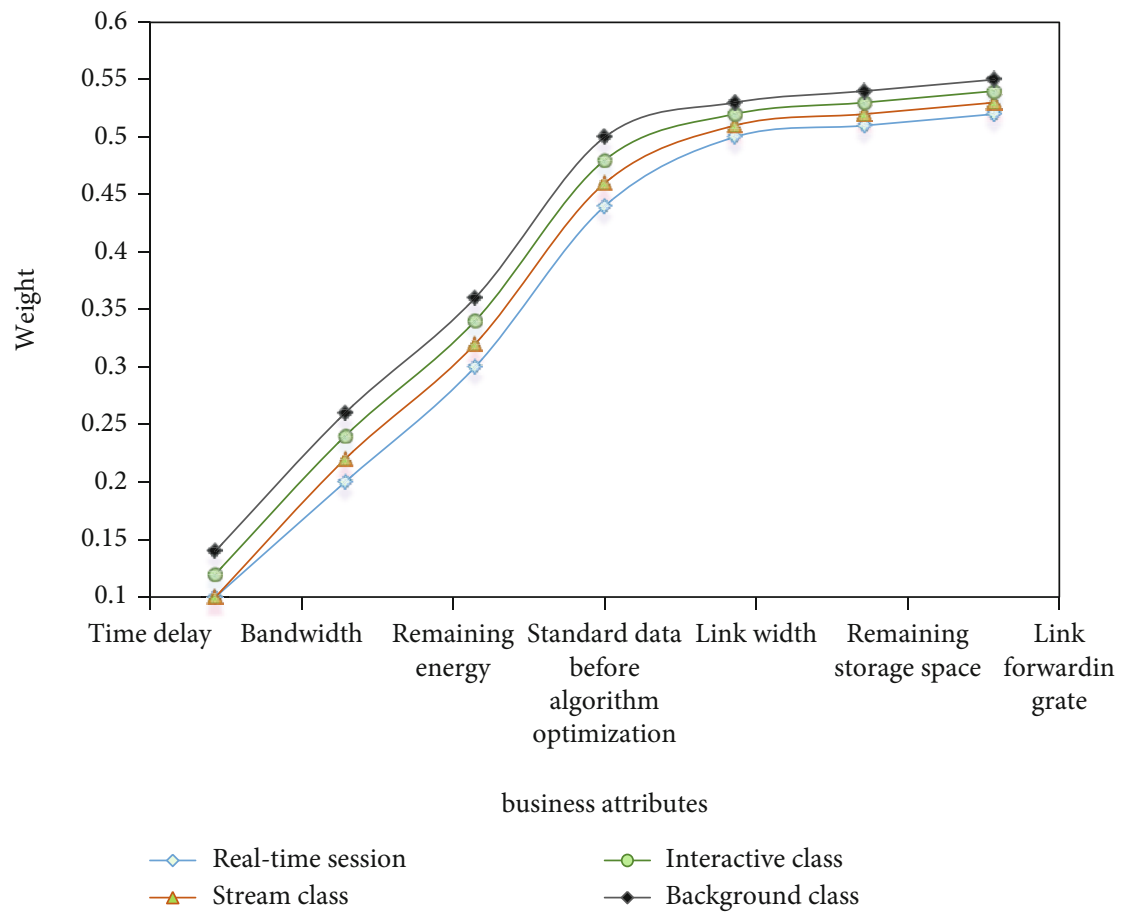

FIGURE 4: Statistic graph of different business weight value parameters.

on DTN routing algorithms. In this experiment, 6 types of services were selected for multiattribute decision-making, the method weight value of spatial DTN network routing optimization was tested, and the data was recorded in Figure 4 below.

The routing algorithm should be able to provide the optimal path from the source end to the destination end, where optimal refers to the design parameters that need to be considered when calculating the path (parameter setting Table 5). In the DTN environment, it generally refers to data delay and data delay, delivery rate and cache occupancy rate, etc. Multiattribute decision-making for network routing optimization is performed to test the weight value, the network characteristics of the spatial DTN are analyzed according to the weight value of each attribute, and the actual satellite network application is monitored. Time delay characteristics, bandwidth, bit error rate, and other aspects are calculated and analyzed, and each attribute of the adjacent node of the spatial DTN network is newly customized. The data shows that after routing optimization by the multiattribute decision-making algorithm, the weight of each attribute is generated in order to ideally adjust, switch between the communication characteristics of various regional networks, and can tolerate long delays within and between the regional networks. This requires that each node or link that may be broken has an available backup way, so that the failure of the main path will not affect the transmission of data services, and the use of background types, dialog types, real-time conversations, etc. has been networked. Optimization can promote more in-depth research in the field of algorithms in these areas.

For general networks under the application of multiattribute decision-making algorithms, we have conducted exper-
TABLE 5: Related parameter setting table.

\begin{tabular}{lc}
\hline Simulation parameters & Value, type \\
\hline Protocol & PROPHET, DTN, ILAR \\
Simulation area (square meter) & $300 * 300$ \\
Number of nodes & $40,50,60,70,80$ \\
Node radius (m) & 200 \\
Simulation time (s) & 100 \\
Channel width & $10 \mathrm{M}$ \\
\hline
\end{tabular}

iments and conducted data statistics. DTN based on multiattribute decision-making can better solve the frequent link interruption, uplink and downlink asymmetry, and long-term in the space network. For data transmission problems in the case of delay, data analysis and comparison are performed on different number of network path modes to demonstrate the performance and interval range of each attribute decision of the spatial DTN network under different paths and obtain the final optimized result and related data recorded in Figure 5.

The experimental data in Figure 5 shows that a single calculation method that relies on itself, that is, the general network and spatial DTN network routing, can exert the optimal choice of each attribute under the condition of a single path, and neither double paths nor multiple paths can satisfy the most optimal choice. The multi-attribute decision-making algorithm in the above figure optimizes the spatial DTN network under different network paths. The final results of the data can be displayed and analyzed. The best values of double paths and multiple paths have 


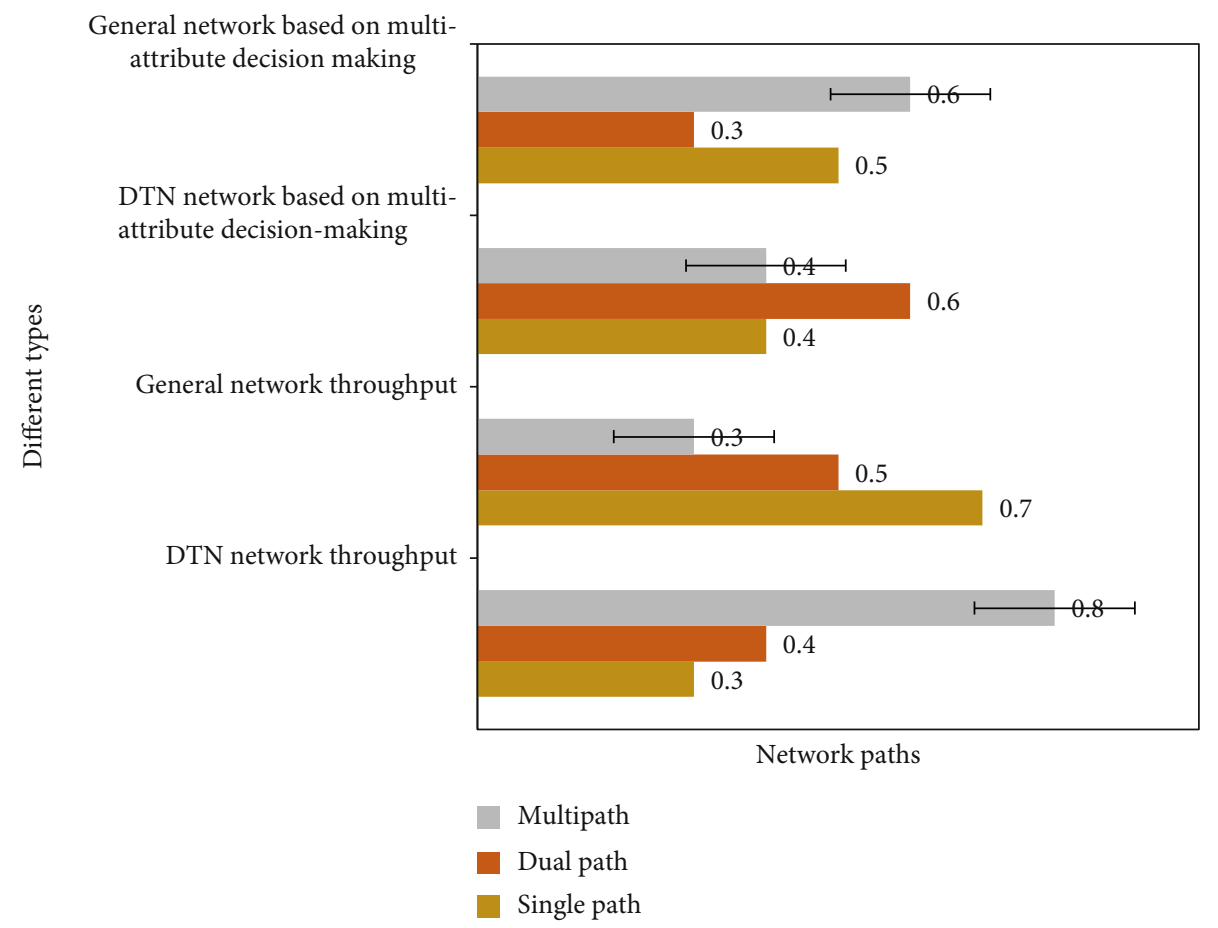

Figure 5: Diagrams of DTN throughput and general network throughput for multiple attribute decision-making with different numbers of network paths.

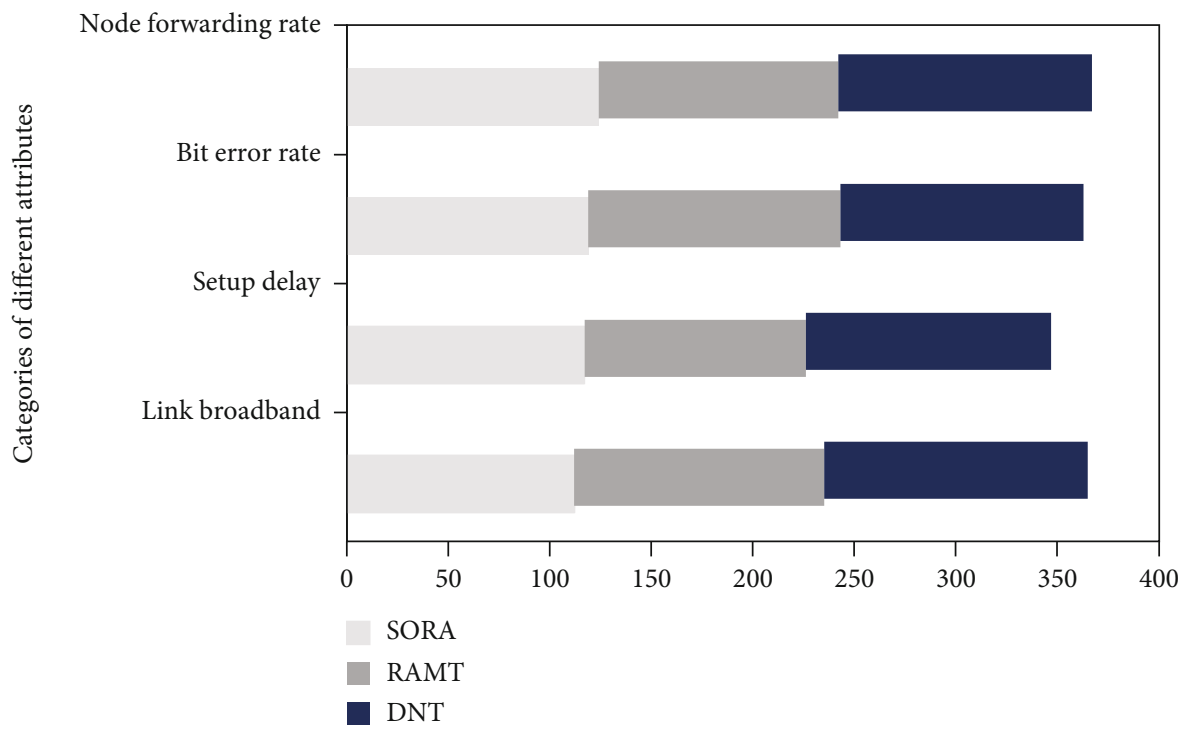

FIGURE 6: The influence of different algorithms on route reconstruction link bandwidth, link establishment delay, network bit error rate, and node data forwarding rate.

been improved and can improve the use of the network system. It can adapt to the rapid changes of the network topology due to the movement of nodes and the limited physical conditions of the equipment and can complete the transmission of data packets in the case of link failures. To a certain extent, it can improve the data transmission performance of the spatial information network.

During the data transmission process, first, transmit on the main path. If the main path fails, transfer the data to the backup path for transmission until both paths fail. In view of the importance of bandwidth measurement technology, many scientific research institutions, universities, academic groups, and enterprises at home and abroad are engaged in the research of bandwidth measurement technology. Although they are still based on end-to-end transmission, in order to solve the problem of data transmission under the conditions of long time delay and frequent interruption of the space link, realizing the real heterogeneous 
network interconnection, we have carried out statistics and recorded in Figure 6.

The data traffic after decomposition is predicted by the spatial DNT network optimized by the network routing. The link dynamic changes in the spatial information network, the propagation time is extended, and the bit error rate is high, that is to say, the factors that cause the increase in transmission delay in the spatial network. It is not only the queuing delay caused by the increase of traffic in the network, but also the difficulty of estimating the available bandwidth. But it solves the problem of data transmission under the conditions of high bit error rate, long delay, uplink and downlink asymmetry, and frequent link interruption in the space network. Moreover, with the rapid development of space applications, the problem of low data transmission efficiency in spatial information networks has become more and more prominent. The application of multiattribute decision-making algorithms can effectively use the application of multiattribute decision-making algorithms to reduce network resources and reduce data transmission delays and error rates.

\section{Conclusion}

Multiattribute decision-making has a wide range of practical applications in society, life, economy, and politics, and it plays an important role. This article introduces the theory of multiattribute decision-making, through the basic principles and decision-making process of multiattribute decisionmaking. The multiattribute decision-making algorithm is applied to the spatial DTN network, which can ensure the dynamic selection of data transmission routes, increase the total amount of returned data, but at the same time reduce the cost and risk of instruction operations, and is more economical than other routing algorithms effective. DTN network application scenarios are changeable and large in scale. The DTN network reliable routing algorithm based on time-varying graph, the service-aware DTN network routing algorithm, and the designed DTN network routing algorithm are simulated in this paper, and the spatial network routing algorithm is built. The simulation test environment verifies the function and performance of the routing algorithm through data transmission and provides theoretical guidance and suggestions for the future construction of the world-earth integrated information network. Node resource limitation is an important attribute in multiattribute decision-making, which affects the overall calculation and technology application of the spatial DTN network. When discussing the sensitivity analysis of multiattribute decision-making methods in the literature, it is more to discuss the influence of the change of attribute weight on the decision result, but it is not only the change of attribute weight that actually affects the order of the decision plan, but the number of decision plans may also has an impact on the sorting, that is, the occurrence of the reverse order phenomenon. This should be paid attention to and discussed in depth. This paper mainly conducts related research on the multiattribute decision-making routing algorithm in the spatial DTN network. It does not analyze the congestion caused by the excessive amount of data in the routing process. The next step requires node-based storage space and network data flow. Analyze the limited node resources of the spatial DTN network.

\section{Data Availability}

No data were used to support this study.

\section{Conflicts of Interest}

The authors declare that there is no conflict of interest with any financial organizations regarding the material reported in this manuscript.

\section{References}

[1] J.-W. Kim, K.-R. Lee, J.-H. Jo, and I. Joe, "Performance modeling of DTN-based file transmission for space internet," Advanced Science Letters, vol. 25, no. 1, pp. 75-78, 2019.

[2] J. Huang, W. Liu, Y. Su, and F. Wang, "Multi-rate combination of partial information-based routing and adaptive modulation and coding for space deterministic delay/disruption tolerant networks," IET Communications, vol. 11, no. 9, pp. 13651370, 2017.

[3] Z. Xu and Z. Cai, "Privacy-preserved data sharing towards multiple parties in industrial IoTs," IEEE Journal on Selected Areas in Communications (JSAC), vol. 38, no. 5, pp. 968-979, 2020.

[4] Z. Cai and Z. He, "Trading private range counting over big IoT data," in The 39th IEEE International Conference on Distributed Computing Systems (ICDCS 2019), 2019.

[5] P. Jacquet, S. Malik, B. Mans, and A. Silva, "On the throughput-delay trade-off in georouting networks," IEEE Transactions on Information Theory, vol. 62, no. 6, pp. 32303242, 2016.

[6] L. Shi, J. Jiao, A. Sabbagh et al., "Integration of Reed-Solomon codes to licklider transmission protocol (LTP) for space DTN," IEEE Aerospace and Electronic Systems Magazine, vol. 32, no. 4, pp. 48-55, 2017.

[7] G. Yu, Z. Dong, and Y. Zhu, "Network evaluation and protocol deployment for complex deep-space networks based on DTN," China Communications, vol. 17, no. 9, pp. 237-258, 2020.

[8] G. Wang, S. C. Burleigh, R. Wang, L. Shi, and Y. Qian, "Scoping contact graph-routing scalability: investigating the system's usability in space-vehicle communication networks," IEEE Vehicular Technology Magazine, vol. 11, no. 4, pp. 46-52, 2016.

[9] Y. Sun, H. Song, A. J. Jara, and R. Bie, "Internet of things and big data analytics for smart and connected communities," IEEE Access, vol. 4, pp. 766-773, 2016.

[10] S. Yang, B. Deng, J. Wang et al., "Scalable digital neuromorphic architecture for large-scale biophysically meaningful neural network with multi-compartment neurons," IEEE transactions on neural networks and learning systems, vol. 31, no. 1, pp. 148-162, 2020.

[11] N. Mizukami, M. P. Clark, K. Sampson et al., "mizuRoute version 1: a river network routing tool for a continental domain water resources applications," Geoscientific Model Development, vol. 9, no. 6, pp. 2223-2238, 2016. 
[12] Y.-C. R. Lai, M. H. Dingler, C.-E. Hsu, and P.-C. Chiang, "Optimizing train network routing with heterogeneous traffic," Transportation Research Record: Journal of the Transportation Research Board, vol. 2159, no. 1, pp. 69-76, 2018.

[13] B. Vignac, F. Vanderbeck, and B. Jaumard, "Reformulation and decomposition approaches for traffic routing in optical networks," Networks, vol. 67, no. 4, pp. 277-298, 2016.

[14] P. Kleer and G. Schafer, "The impact of worst-case deviations in non-atomic network routing games," Theory of Computing Systems, vol. 63, no. 1, pp. 54-89, 2019.

[15] Y. Yang, M. Xu, D. Wang, and Y. Wang, "Towards energyefficient routing in satellite networks," IEEE Journal on Selected Areas in Communications, vol. 34, no. 12, pp. 38693886, 2016.

[16] B. S. Kushwaha and P. K. Mishra, "Different traffic patterns over ad hoc network routing protocols," International Journal of Computer Applications, vol. 138, no. 11, pp. 1-5, 2016.

[17] D. J. Persis and T. P. Robert, "Reliable mobile ad-hoc network routing using firefly algorithm," International Journal of Intelligent Systems Technologies \& Applications, vol. 8, no. 5, pp. 10-18, 2016.

[18] F. Nawaz and V. Jeoti, "GREEN protocol: gradient based energy efficient routing with network coding capacity," Telecommunication Systems, vol. 62, no. 1, pp. 135-147, 2016.

[19] G. Lin, S. Soh, K.-W. Chin, and M. Lazarescu, "Power-aware routing in networks with quality of services constraints," Transactions on Emerging Telecommunications Technologies, vol. 27, no. 1, pp. 122-135, 2016.

[20] M. Al, "Mobile ad hoc network routing protocols: performance evaluation and assessment," IJCDS Journal, vol. 7, no. 1, pp. 59-66, 2018.

[21] H. Prasad and S. Babu, "A survey on network routing protocols in internet of things (IOT)," International Journal of Computer Applications, vol. 160, no. 2, pp. 18-22, 2017.

[22] K. Pothuganti, "Overview of vehicular adhoc network routing protocols and their architecture," International Journal of Innovative Research in Computer and Communication Engineering, vol. 8, no. 9, pp. 3708-3711, 2020.

[23] A. H. Ulusoy, "Investigation of delay tolerant network routing protocols with energy consumption analysis," Ad Hoc \& Sensor Wireless Networks, vol. 46, no. 1-2, pp. 53-82, 2020.

[24] E. Rao, "Wireless sensor network routing for life time maximization using ANFIS based decision with low power consumption," Turkish Journal of Computer and Mathematics Education (TURCOMAT), vol. 12, no. 2, pp. 2893-2900, 2021.

[25] A. Begum, Z. R. Zahid, and N. Nancy, "Comparative performance evaluation of mobile ad-hoc network routing protocols using NS2 simulator," International Journal of Recent Technology and Engineering, vol. 9, no. 3, pp. 707-713, 2020.

[26] T. Yélémou, B. Zerbo, and T. M. Dandjinou, "Analysis of adhoc network routing metrics efficiency," Journal of Theoretical and Applied Information Technology, vol. 98, no. 23, pp. 38673878, 2020.

[27] H. Zemrane, Y. Baddi, and A. Hasbi, "Mobile ad hoc network routing protocols for intelligent transportation systems,"
International Journal of Smart Security Technologies, vol. 8, no. 1, pp. 35-48, 2021.

[28] A. Arslan, "Computer network routing configuration based on intelligent algorithm," Acta Electronica Malaysia, vol. 4, no. 1, pp. 28-30, 2020.

[29] Y. Liu and Y. Morgan, "Rate region of unicast routing networks," Electronics Letters, vol. 52, no. 21, pp. 1765-1767, 2016. 